\title{
A possible means of monitoring the progress of demyelination in multiple sclerosis: effect of body temperature on visual perception of double light flashes
}

\author{
R. J. GALVIN, D. REGAN, AND J. R. HERON \\ From the Department of Neurology and Medical Research Unit, North Staffordshire Hospital Centre, \\ Stoke-on-Trent, and the Department of Communication, University of Keele, Staffordshire
}

SYNOPSIS The ability to discriminate closely separated pairs of light flashes as being double is impaired in multiple sclerosis. The effects of altering body temperature on double flash resolution and on visual acuity were studied in four multiple sclerosis patients and in control subjects. At demyelinated sites heating impaired and cooling improved double flash resolution. Visual acuity behaved similarly. The double flash test was very sensitive, changing up to $75 \mathrm{~ms}$ in response to simple heating and cooling procedures that produced small variations in acuity. Apart from its diagnostic value, the double flash test furnishes a simple in vivo model to study the effect of temperature change (and potential symptomatic therapy) on conduction in partially demyelinated axons in the visual system.

It is well known that alterations in body temperature can markedly change the signs and symptoms of multiple sclerosis. Some patients show a dramatically large effect; sensitivity may be so high that circadian temperature changes produce clear periodic variation in visual acuity (Namerow, 1968) and motor function (Davis et al., 1973). Uhthoff (1890) was the first to describe transient blurring of vision as a symptom of multiple sclerosis. It may be caused by physical exertion or a warm bath. Increasing body temperature may extend scotomata and reduce visual acuity (Namerow, 1968), raise critical flicker frequency (Namerow, 1971), and worsen motor signs (Davis, 1966). On the other hand, decreasing body temperature can reverse the impairments of visual and motor function (Watson, 1959). Changes in calcium ion concentration produced by oral ingestion of phosphate can also reverse impairment of visual and oculomotor function (Becker et al., 1974).

Experimental demyelination in animal preparations has been shown markedly to impair or even block conduction in single nerve fibres in the

(Accepted 16 April 1976.)
CNS (McDonald and Sears, 1970). Reversible blockage of conduction has been shown to occur in response to temperature change of as little as $0.5^{\circ} \mathrm{C}$, across identifiable internodes of single ventral root fibres in the rat (Rasminsky, 1973). The effect of temperature (and also the effects of calcium ion concentration and $\mathrm{pH}$ ) upon conduction along partially demyelinated axons is well understood in terms of known axonal physiology even at a quantitative level (Hodgkin and Huxley, 1952; Koles and Rasminsky, 1972; Davis and Schauf, 1974). With minor modifications (Davis and Schauf, 1974) the HodgkinHuxley model not only casts light on the pathophysiology of multiple sclerosis and explains some discrepancies between the physical signs and the pathological changes but can also suggest approaches to the search for symptomatic therapy.

The salient point is that, for a population of partially-demyelinated axons, increasing body temperature mimics the effect of increased demyelination, while decreasing body temperature mimics the effect of decreased demyelination (Davis and Schauf, 1974).

When a multiple sclerosis patient with overt or 
subclinical optic neuropathy views a pair of brief light flashes the interval between them must be considerably longer than for normal control subjects if he is to see them as double (Galvin et al., 1976). This double flash test is simple, sensitive enough to detect subclinical demyelination in the visual pathway, and has proved to be an aid to diagnosis. We report here that the double flash test provides an easily measured index of temperature sensitivity in the partially demyelinated visual pathway.

\section{METHODS}

DOUBLE FLASH DISCRIMINATION The subjects viewed a small (20 minutes of arc subtense) solid state red lamp that gave a pair of brief flashes separated by a variable dark interval. The lamp was surrounded by a diffuse white surface illuminated to a level of $54.5 \mathrm{~cd} / \mathrm{m}^{2}$ (1.2 log ft lamberts) in order to maintain the eye at a daylight (photopic) level of light adaptation. The shortest interval between a pair of flashes for which they could be seen as double was measured by the procedure of ascending and descending limits (Kling and Riggs, 1972) as follows. The subject was instructed to report either a single or double flash for each stimulus presentation. Starting with a large interflash interval giving a stimulus confidently reported as double a series of flash pairs was delivered decreasing the interval between flashes by $5 \mathrm{~ms}$ each time. This was continued until the subject reported a single flash. Threshold was defined as lying midway between the last stimulus and the immediately preceding one. The next series was started well below this threshold with a very short interflash giving a stimulus confidently reported as a single flash. The interval between the flashes was then increased in 5 ms steps until a stimulus was reached where the subject changed from a response of single to double. The ascending threshold was calculated as lying midway between this last stimulus and the immediately preceding one. Two ascending and two descending series were performed and a mean double flash threshold calculated.

VISUAL ACUITY The subject set the separation of two fine vertical lines on a cathode ray oscilloscope so that they could just be seen as double (Michael and Davis, 1973). Visual acuity was measured as the angular distance between the lines.

COOLING PROCEDURE The subjects ingested $300 \mathrm{ml}$ finely crushed ice flavoured with lime juice and then immersed both hands in iced water. This cooling procedure was restricted to less than 10 minutes.
WARMING PROCEDURE Both legs were immersed to mid-shin level in water at $44^{\circ} \mathrm{C}$ and the subject was wrapped in blankets with three domestic hot water bottles around the abdomen. Warming was continued until a clear change in double flash interval was noted at demyelinated sites and for $\mathbf{4 5}$ minutes in the control series.

PATIENTS Four multiple sclerosis patients with clinical optic nerve involvement were studied. Heating was carried out in three and cooling in three.

Patient 1, a woman aged 38 years, had definite multiple sclerosis according to the criteria of McAlpine et al. (1968). She had bilateral optic atrophy. A cooling experiment was carried out in her right eye which had been the site of classical retrobulbar neuritis 17 months previously. Possible temperature sensitivity was indicated by a complaint of intensified leg paraesthesiae in warm weather.

Patient 2, a woman aged 31 years, had definite multiple sclerosis including previous episodes of left and right retrobulbar neuritis. Heating and cooling experiments were carried out in her right eye which had been affected by classical retrobulbar neuritis six years previously without subsequent optic atrophy. She was clinically temperature sensitive with marked deterioration in leg power on warm bathing but without accompanying visual disturbance.

Patient 3, a man aged 29 years, had definite multiple sclerosis including bilateral optic atrophy. Heating and cooling experiments were performed at an extrafoveal site of abnormal double flash resolution in his right eye. This eye had been affected by classical retrobulbar neuritis eight years previously. He had noticed a mild increase in weakness but no visual disturbance on warm bathing.

Patient 4, a woman aged 42 years, had a progressive dorsal (D9) cord lesion for 10 months. There was no history of optic neuritis but for six months she had a striking right Uhtoff symptom with blurring of vision on exertion and marked deterioration of vision on taking a warm bath. There was no pallor of optic discs but a reduced number of small vessels crossing the right disc margin. The left optic disc was normal.

CONTROL MEASUREMENTS Control experiments were performed six times, three heating and three cooling.

1. One heating and two cooling experiments were performed in normal volunteer subjects.

2. A heating experiment was performed in the clinically normal left eye of multiple sclerosis patient 4. 
3. One cooling and one heating series were performed in the right eye of patient 3 as the fovea at this site displayed normal double flash resolution and acuity. Some extrafoveal sites in this eye displayed abnormal double flash resolution.

\section{RESULTS}

The overall results of this study are illustrated by Fig. 1. Panels a, b and c show results from multiple sclerosis patient 2 . Heating (a) degraded double flash resolution and there was a deterioration in acuity that correlated with the rise in double flash threshold. Conversely, on cooling (c) double flash resolution and visual acuity improved. The central set of readings (b) was carried out without heating or cooling and demonstrates that no change in double flash resolution or visual acuity occurred as a result of

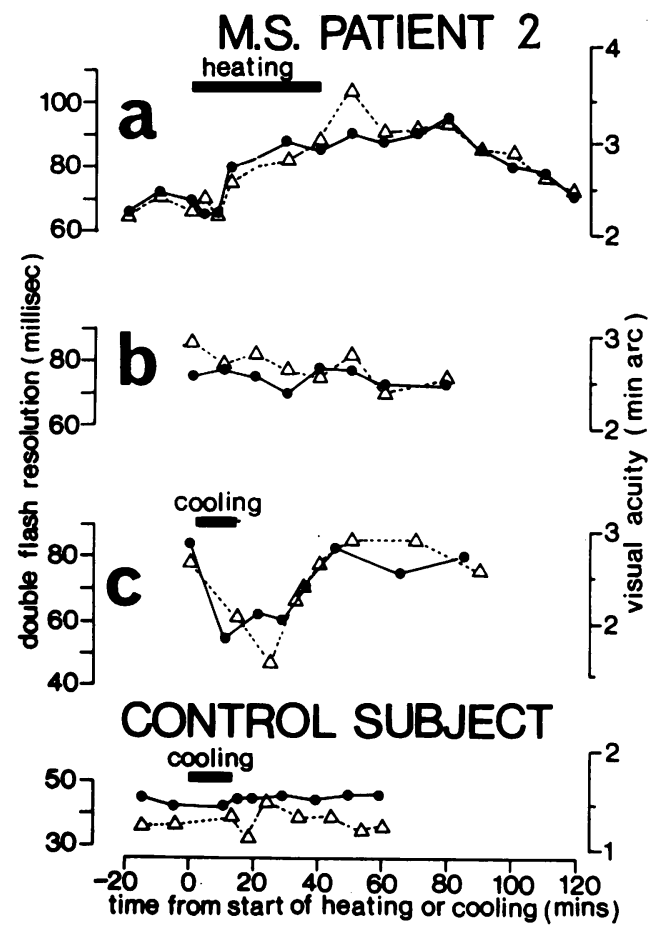

FIG. 1 Alterations in double flash resolution ( -0$)$ and visual acuity $(\triangle-\cdots \triangle)$ in response to temperature change in multiple sclerosis patient $2(a, b$, and $c)$ and a control subject. the experimental procedure itself. The bottom trace shows that cooling in a control subject produced no significant change in double flash resolution (less than \pm 2 ms range in this case) or in visual acuity (less than \pm 0.2 min arc range in this case).

Figure 2 shows a striking deterioration in double flash resolution produced by heating the patient with Uhthoff's symptom. This patient's unaffected left eye acted as her own precise control, as local body temperature was presumably the same for both optic nerves. In the affected eye the change in double flash resolution relative to the change in acuity was considerably greater than that shown by the patient in Fig. 1 .

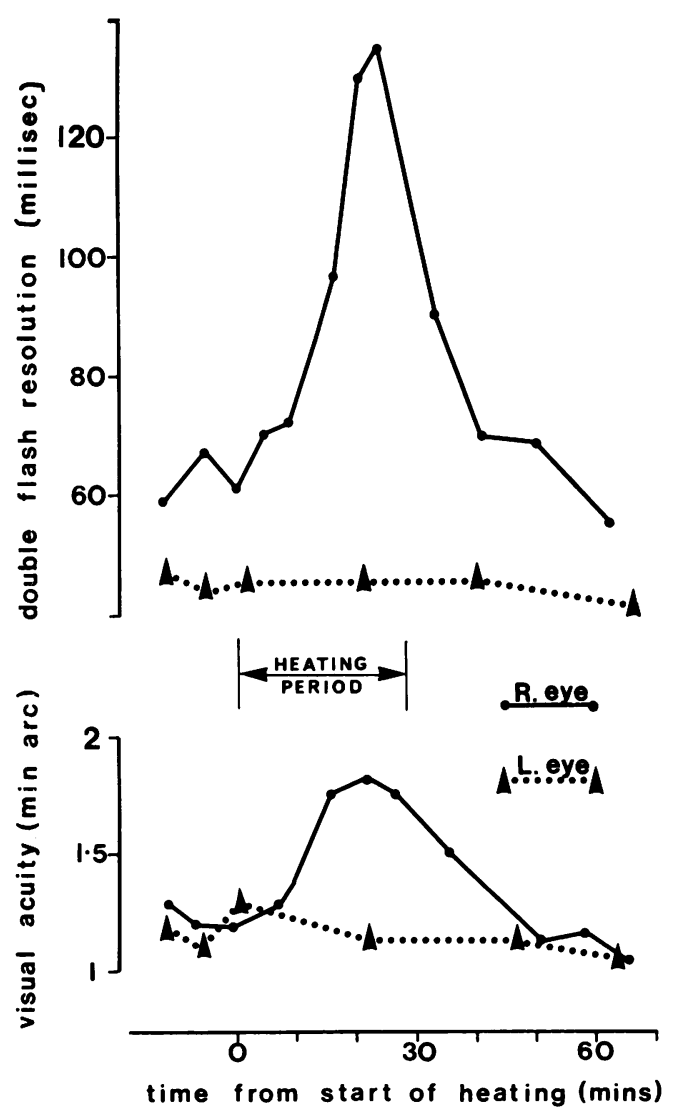

FIG. 2 The effect of heating on double flash resolution and visual acuity in the affected and unaffected eyes of a patient with right-sided Uhthoff symptom. 
Patient 3 showed patchy impairment of double flash resolution within the right central visual field. This type of impairment, due to localized 'islands of demyelination', has been reported previously (Galvin et al., 1976). A site of normal double flash resolution (at the fovea) and a site of abnormal double flash resolution $\left(4^{\circ}\right.$ eccentric to the fovea) were studied simultaneously. Figure 3 illustrates the effect of cooling on these two sites. At the eccentric site (A) of abnormal double flash threshold there was a prompt and marked fall $(30 \mathrm{~ms})$ in double flash threshold to normal with a less pronounced and more short lived improvement in acuity. At the central site (B) of normal double flash resolution, cooling did not produce any definite changes in double flash resolution or acuity. Heating this patient produced a slight deterioration in double flash threshold (10 ms), but no acuity change, at the affected site.

The values obtained from all the abnormal and control series are presented in the Table.

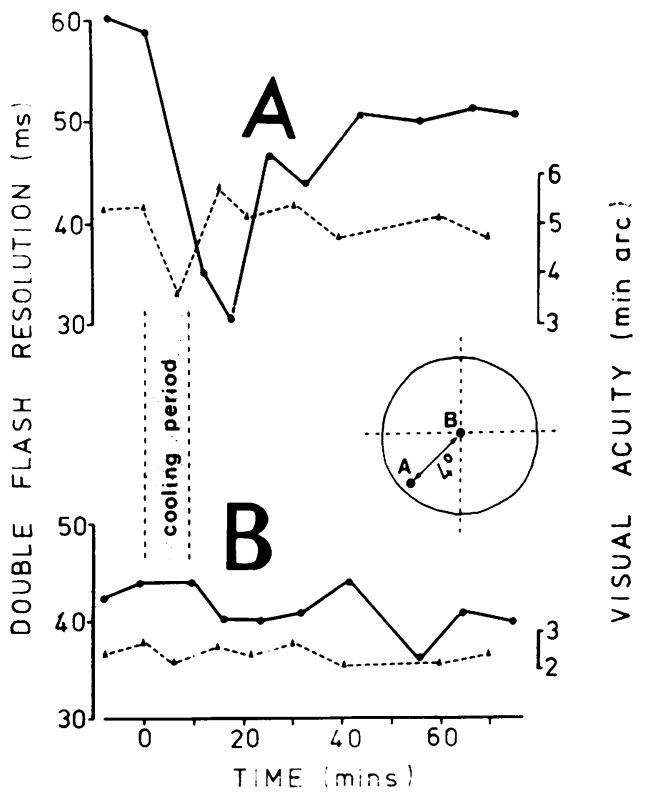

FIG. 3 After retrobulbar neuritis this patient's right eye displayed abnormal double flash resolution at site $A, 4^{\circ}$ eccentric to the fovea where resolution was normal (site B). Cooling reverses the abnormality at site A. - = double flash. - . - - = acuity.
TABLE

CHANGES IN DOUBLE FLASH RESOLUTION AND VISUAL ACUITY FROM ALL DEMYELINATED AND NORMAL SITES TESTED

\begin{tabular}{lccc}
\hline Subject & $\begin{array}{c}\text { Heating or } \\
\text { cooling }\end{array}$ & $\begin{array}{c}\text { Max. rise/fall in } \\
\text { D-F resolution } \\
(\text { ms })\end{array}$ & $\begin{array}{c}\text { Max. rise/fall in } \\
\text { acuity }(\text { min arc })\end{array}$ \\
\hline MS & C & 18 & 0.8 \\
MS & C & 30 & 1.1 \\
MS & C & $30^{*}$ & $1.8^{*}$ \\
MS & H & 29 & 1.4 \\
MS & H & $10^{*}$ & $0.1^{*}$ \\
MS & H & 75 & 0.7 \\
Normal & C & 2 & 0.2 \\
Normal & C & 4 & 0.1 \\
MS control & C & 6 & 0.3 \\
Normal & H & 0 & 0.1 \\
MS control & H & 2 & 0.2 \\
MS control & H & 1 & 0.2 \\
\hline
\end{tabular}

*Measured $4^{\circ}$ eccentric to the fovea.

\section{DISCUSSION}

We report here that a simple double flash test is capable of measuring a response to temperature change in patients with optic neuropathy. Cooling the patient improves double flash discrimination while heating degrades discrimination. No effect was observed in control series. When changes in visual acuity were large enough to detect, they concurred with our double flash measurements, both showing the same paradoxical response to temperature change. It should be noted that Michael and Davis (1973) produced much greater changes in visual acuity in their multiple sclerosis patients than we have presented in this paper. They, however, heated their patients in a steam cabinet to raise body temperature as a whole by as much as $3^{\circ} \mathrm{C}$, whereas we utilized much less stressful heating and cooling procedures that presumably gave only local and temporary changes in temperature. These procedures giving small changes in acuity produced large alterations in double flash resolution. A rise of $75 \mathrm{~ms}$ is equivalent to a change of 20 standard deviations from our normal range (Galvin et al., 1976).

The provoking effect of elevated body temperature in reducing visual acuity (and other symptoms and signs) has been used to aid the diagnosis of multiple sclerosis (Davis, 1966). We have reported previously that in many patients the double flash test is a more sensitive measure of 
demyelination than changes in visual acuity (Galvin et al., 1976). This suggests that the double flash test may offer enhanced sensitivity in such diagnostic procedures.

Although neither changes in body temperature nor changes in calcium ion concentration offer methods of practical symptomatic therapy, recent advances in understanding of conduction impairment have led to suggestions that pharmacological agents might, by prolonging the action potential duration, improve conduction in demyelinated nerve fibres and thus have therapeutic value in multiple sclerosis (Davis et al., 1970). If the effects of decreasing body temperature provide a realistic model of improved conduction in a population of partially demyelinated axons, then the double flash test might offer a means of objectively assessing the effectiveness of such experimental therapies for multiple sclerosis.

We thank the Multiple Sclerosis Society for a research fellowship to one of us (R.J.G.). Wilkinson Sword Research developed and supplied the double-flash device.

\section{REFERENCES}

Becker, F. O., Michael, J. A., and Davis, F. A. (1974). Acute effects of oral phosphate on visual function in multiple sclerosis. Neurology (Minneap.), 24, 601-607.

Davis, F A. (1966). Hot bath test in the diagnosis of multiple sclerosis. Journal of Mount Sinai Hospital, 33, 280-282.

Davis, F. A., Becker, F. O., Michael, J. A., and Sorensen, E. (1970). Effect of intravenous sodium bicarbonate, disodium edetate ( $\mathrm{Na}_{2}$ EDTA), and hyperventilation on visual and oculomotor signs in multiple sclerosis. Journal of Neurology, Neurosurgery, and Psychiatry, 33, 723-731.

Davis, F. A., Michael, J. A., and Tomaszewski, J. S. (1973). Fluctuation in motor function in multiple sclerosis related to circadian temperature variations Diseases of the Nervous System, 34, 33-36.
Davis, F. A., and Schauf, C. L. (1974). The pathophysiology of multiple sclerosis: a theoretical model. In Models of Human Neurological Disease. Edited by H. J. Klawans, Jr. Excerpta-Medica: Amsterdam.

Galvin, R. J., Regan, D., and Heron, J. R. (1976). Impaired temporal resolution of vision after acute retrobulbar neuritis. Brain, 99, 255-268.

Hodgkin, A. L., and Huxley, A. F. (1952). A quantitative description of membrane current and its applications to conduction and excitation in nerve. Journal of Physio$\log y, 117,500-544$.

Kling, J. W., and Riggs, L. A. (1972). (eds). Woodworth and Schlosberg's Experimental Psychology, pp. 14-20. Methuen: London.

Koles, Z. J., and Rasminsky, M. (1972). A computer simulation of conduction in demyelinated nerve fibres. Journal of Physiology, 227, 351-364.

McAlpine, D., Lumsden, C. E., and Acheson, E. D. (1968). Multiple Sclerosis, Reappraisal, pp. 183-184. Livingstone: Edinburgh.

McDonald, W. I., and Sears, T. A. (1970). The effects of experimental demyelination in the central nervous system. Brain, 93, 583-598.

Michael, J. A., and Davis, F. A. (1973). Effects of induced hyperthermia in multiple sclerosis: differences in visual acuity during heating and recovery phases. Acta Neurologica Scandinavica, 49, 141-151.

Namerow, N. S. (1968). Circadian temperature rhythm and vision in multiple sclerosis. Neurology (Minneap.), $18,417-422$.

Namerow, N. S. (1971). Temperature effect on critical flicker fusion in multiple sclerosis. Archives of Neuro$\log y, 25,269-275$.

Rasminsky, M. (1973). The effects of temperature on conduction in demyelinated single nerve fibers. Archives of Neurology (Chic.), 28, 287-292.

Uhthoff, W. (1890). Untersuchungen über die bie der multiplen herdsklerose vorkommenden augenstörungen. Archiv für Psychiatrie und Nervenkrankheiten, 21, 55-116, 303-410.

Watson, C. W. (1959). Effect of lowering of body temperature on the symptoms and signs of multiple sclerosis. New England Journal of Medicine, 261, 1253-1259. 